ing fieldwork acceptable was so great that he acquired the nickname that gives the present book its title.

Griscom coupled his enormous enjoyment of birds with a strong sense of the need for conservation and played an important part in its development in the United States. The last years of his life were dogged by ill-health, mainly bad circulation, apparently as a result of heavy smoking - a habit he refused to give up. His condition lead to a series of strokes, the first in 1950, but he battled on, continuing to work until almost the end.

This book is a useful, thoroughly researched account of the life and work of this dynamic and forceful man, with whom it was apparently not always easy to get along. It contains a valuable appendix to all his published works.

C. M. Perrins is in the Department of Zoology, University of Oxford, South Parks Road, Oxford OX1 3PS, UK.

\section{Limits to knowledge}

\section{Adam Schultz}

Geophysical Inverse Theory. By Robert L. Parker. Princeton University Press: 1994. Pp. 386. £30, \$39.50.

IT is comforting to some that the realm of quantum theory typified by Heisenberg's uncertainty principle can be placed in a box and neatly ignored by those concerned with phenomena on the scale of everyday experience. That modern inverse theory breaks down this artificial barrier, and extends the notion that there are intrinsic limits to knowledge on all scales, may prove disturbing, yet it stands as one of the most important achievements of mathematical physics.

Inverse theory provides a framework for solving the inverse problem: that is, determining the values of the parameters that describe a physical model given a set of measurements sensitive to these parameters. This generally also requires solving a forward problem - determining the values the measurements would have, given a model with a set of known values of the parameters. Solving the inverse problem is generally a much more difficult proposition than solving the forward problem, and the best one can usually hope for is to place bounds on the unknown parameters, rather than to solve for them uniquely.

An example of a forward problem is to determine the seismic waveforms on the surface of the Earth given a model of the Earth's elastic parameters and a known seismic source. The corresponding inverse problem is to recover these elastic parameters given a set of observations of earthquakes on the Earth's surface.

The relationship between the unknown parameters and the observed data is often nonlinear and the data are typically inadequate and contaminated by noise. Inverse models are usually nonunique: an infinite number of different models may lead to exactly the same set of observations. This may be due to the underlying physics or to the inexactness and sparsity of observations.

This is a field where many different schools contend. Robert Parker's inverse theory is less statistical than, say, Albert Tarantola's, and Geophysical Inverse Theory is a view of the field from Parker's particular perspective rather than a survey of the entire spectrum of thought.

The student of inverse theory must begin with a foundation in functional analysis. Parker builds on the work of pioneering inverse theoreticians such as George Backus and Freeman Gilbert, who in the 1960 s built a sound mathematical basis for geophysical inversion, and placed inverse theory in terms of linear vector spaces (for example, Hilbert space). One of Parker's most valuable roles has been to interpret the sometimes opaque mathematics of these classical works and to express them in terms that are understandable to the numerate nonmathematician.

After a tutorial on vector spaces, Parker introduces linear inverse theory and then considers the effects of uncertain data (noise) on the resulting inverse models. It is here that formulation of the inverse problem in terms of Hilbert space is particularly convenient.

Parker provides a clear description of linear resolution theory, and points out shortcomings in the classical Backus-Gilbert theory. A well-considered section on means of bounding linear functionals is followed naturally enough by ideal body theory, Parker's most important contribution to potential fields inversion (for example, gravitational and magnetic), and also by linear and quadratic programming, the methods by which such problems may be solved.

There is a brief discussion of a 'statistical theory' of inversion, but readers would do well to look elsewhere for a more detailed view of this approach. The final chapter is devoted to nonlinear inverse problems (most real-world problems are nonlinear) with particular emphasis on the mildly nonlinear magnetotelluric problem of finding the Earth's electricalconductivity structure.

There is no general theory of nonlinear inversion; the usual approach is to reformulate the problem in linear terms around a starting model, and to solve it using an iterative process. Parker is a leading authority on this approach, and the final chapter is an excellent, although brief, introduction.

One might hope for an additional chapter in future editions touching on recent developments in nonlinear optimization theory, such as simulated annealing, genetic and evolutionary algorithms and the like. With this said, however, Parker's work is essential for any student of inverse theory and should be required reading for anyone in the field.

Adam Schultz is in the Institute of Theoretical Geophysics, Department of Earth Sciences, Downing Street, University of Cambridge, Cambridge CB2 3EQ, UK.

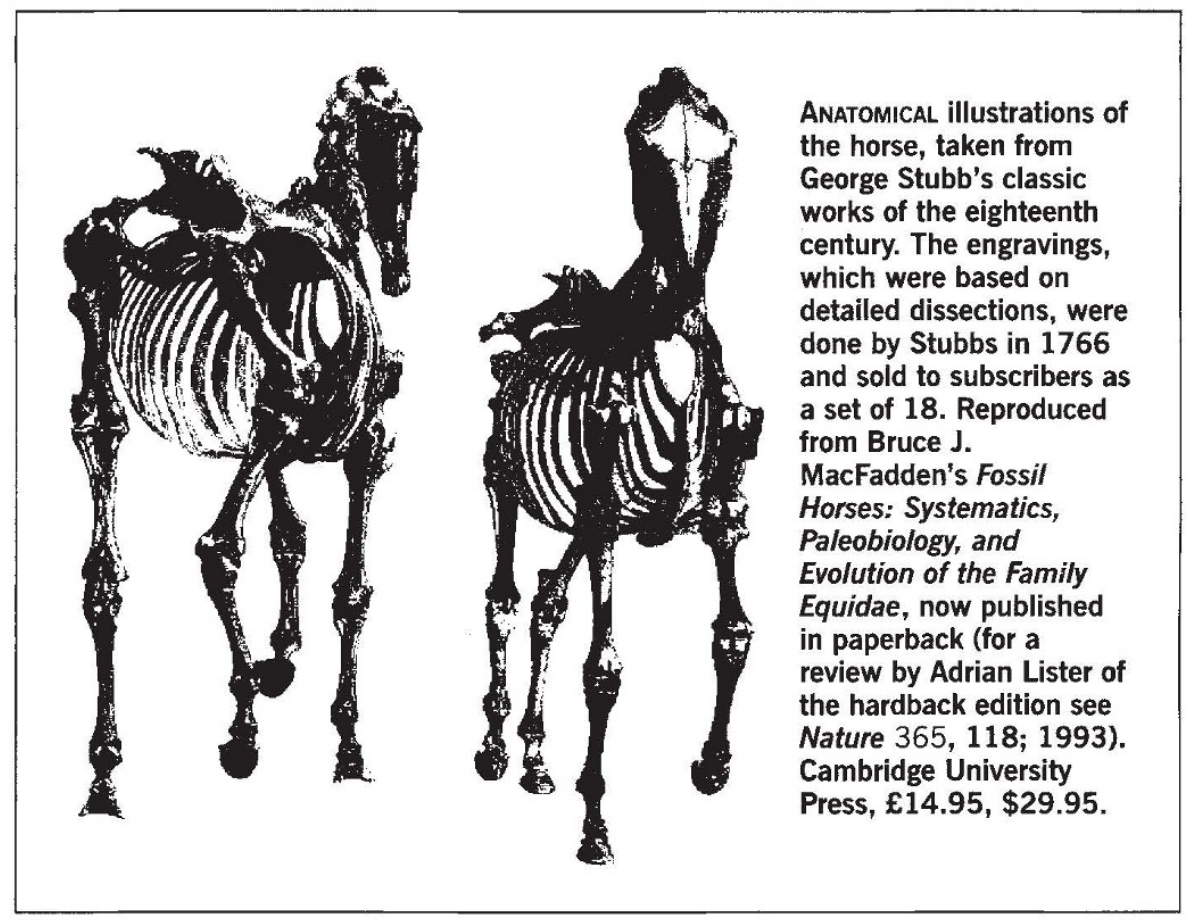

